\title{
Conditioned Pain Modulation in Patients With Acute and Chronic Low Back Pain
}

\author{
Sabine Mlekusch, MD, ${ }^{*}$ Alban Y. Neziri, MD, PhD, *ै \\ Andreas Limacher, MD, PhD, $\$$ Peter Jüni, MD, \\ Lars Arendt-Nielsen, MD, PhD, $\S$ and Michele Curatolo, $M D, P h D \S \|$
}

\begin{abstract}
Objectives: Disturbed endogenous pain modulation is likely one of the mechanisms underlying central hypersensitivity and might be a contributing factor for the development and maintenance of chronic pain. To our knowledge, no study has investigated endogenous pain modulation in both acute and chronic low back pain (LBP). We tested the hypothesis that endogenous pain inhibition is impaired in patients with acute and chronic LBP.
\end{abstract}

Materials and Methods: We evaluated 40 patients with acute LBP, 34 patients with chronic LBP and 30 pain-free controls for their conditioned pain modulation (CPM), with pressure pain tolerance and cold pressor as test and conditioning stimulus, respectively. Measurements were repeated up to 10 minutes after cold pressor test.

Results: There was no difference in CPM among the groups immediately after cold pressor test. However, the decline in CPM effect was significantly faster in chronic and acute LBP patients than in controls, with no evidence for differences between pain groups.

Discussion: The present study provides evidence for some alterations of endogenous modulation in both acute and chronic LBP. CPM was still detected in both patient groups, indicating that endogenous modulation, although effective for a shorter duration, is partially functioning in patients with LBP.

Key Words: conditioned pain modulation, acute low back pain, chronic low back pain

(Clin J Pain 2016;32:116-121)

Received for publication June 18, 2014; revised April 24, 2015; accepted March 12, 2015.

From the *Department of Anesthesiology and Pain Therapy, Inselspital-University Hospital of Bern; $₫$ CTU Bern, Department of Clinical Research, and Institute of Social and Preventive Medicine (ISPM), University of Bern, Bern; †Department of Obstetrics and Gynaecology, Cantonal Hospital of Winterthur, Winterthur, Switzerland; §Department of Health Science and Technology, Center for Sensory-Motor Interaction, University of Aalborg, Aalborg, Denmark; and $\|$ Department of Anesthesiology and Pain Medicine, University of Washington, Seattle, WA.

S.M. and A.Y.N. contributed equally.

Supported by the Swiss National Science Foundation (3247BO_122358/1), Danish Research Council for Technology and Production, Copenhagen, Denmark, Scientific Funds of the University Department of Anesthesiology and Pain Therapy of the University of Bern, and the Foundation for Research in Anesthesia and Intensive Care of the University Hospital of Bern, Bern, Switzerland, CTU Bern is supported by the Swiss National Science Foundation, Bern, Switzerland. The authors declare no conflict of interest.

Reprints: Sabine Mlekusch, MD, Department of Anesthesiology and Pain Therapy, Inselspital-University Hospital of Bern, Bern 3010, Switzerland (e-mail: sabine.mlekusch@insel.ch).

Copyright (C) 2015 Wolters Kluwer Health, Inc. All rights reserved.

DOI: $10.1097 /$ AJP.0000000000000238
$\mathrm{T}$ he central nervous system is capable to modulate signals coming from peripheral tissues, leading to either amplification or attenuation of the nociceptive input. ${ }^{1}$ One important aspect of central pain modulation is endogenous inhibition, as its impaired efficiency may be a determinant of symptoms in pain patients. In humans, 1-test paradigm to evaluate the endogenous pain modulatory system is conditioned pain modulation (CPM), which occurs when the response to a painful test stimulus is inhibited by an additional conditioning painful stimulus. ${ }^{2}$

Disturbed CPM is likely one of the mechanisms underlying central hypersensitivity and might be 1 contributing factor for the development and maintenance of chronic pain. Patients with chronic low back pain (LBP) display decreased pain thresholds after stimulation of nonpainful tissues and enlargement of the areas of referred pain, ${ }^{3-5}$ which is strongly suggestive for widespread central hypersensitivity. Although hypersensitivity has been documented, the mechanisms underlying exaggerated pain responses in acute and chronic LBP are unclear. One of these mechanisms may be deficient endogenous pain modulation. Most studies have focused on chronic pain conditions, but deficient pain modulation could also be present in the acute phase. Furthermore, most studies have assessed CPM at 1 time-point, and information on its time course after cessation of the conditioning stimulus is sparse.

The hypothesis of this study was that endogenous pain inhibition is impaired in patients with acute and chronic LBP. We evaluated the inhibitory effect not only immediately after the conditioning stimulus, but also 3, 5, and 10 minutes after its cessation. The results are expected to improve our knowledge on the role of central modulatory mechanisms in the pathophysiology of LBP and possibly explore mechanisms involved in the transition from acute to chronic pain.

\section{MATERIALS AND METHODS}

\section{Study Population}

Consecutive patients with acute LBP were asked to participate in the study at the mediX practice network of Bern, Switzerland. These patients were part of a still running prospective cohort study on acute LBP. During the same period of time, consecutive patients with chronic LBP were recruited at the Pain Division of the Department of Anesthesiology and Pain Therapy of the University Hospital of Bern, Switzerland. These patients were part of a case-control study. ${ }^{6}$ Pain-free controls were recruited at the University of Bern by advertisement. The period of data acquisition was from January 1, 2009 until October 31, 2011. 
Inclusion criteria for the acute LBP group were pain lasting for no longer than 4 weeks and LBP at the time of testing with an intensity $\geq 3$ using a $10 \mathrm{~cm}$ visual analog scale (VAS), whereby $0=$ no pain and $10=$ worst pain imaginable. Exclusion criteria were any history of chronic LBP and the presence of any type of chronic pain. Inclusion criteria for the chronic LBP group were daily LBP for at least 6 months duration and LBP at the time of testing with an intensity of VAS $\geq 3$. Exclusion criterion was the presence of any other type of chronic pain. For the pain-free controls the inclusion criterion was absence of any acute or chronic painful condition.

Exclusion criteria for all groups were defined as inability to understand the tests, lacking knowledge of German language, radicular pain (as defined by leg pain associated with an MRI finding of a herniated disk or foraminal stenosis with contact to a nerve root), neurological conditions potentially affecting sensory function (ie, polyneuropathy, diabetes mellitus, or alcohol abuse), pregnancy (ruled out by pregnancy test), breast-feeding, intake of oral contraceptives or hormones, intake of opioids and antidepressants during the previous 2 weeks, and intake of other analgesics or drugs known to modulate pain during 48 hours before testing.

Age, sex, and body mass index were recorded for descriptive purposes. The studies were approved by the local ethics committee (KEK no. 103/2008-107/04). Patients and controls gave written informed consent.

\section{Assessments of CPM}

\section{General Aspects of Assessments}

The body side with the higher intensity of pain was determined in patients who had unequal pain intensity on the 2 body sites, and was chosen for the application of pressure stimulation. In case of bilateral pain of equal intensity and in the control group, the side of application of pressure stimulation was randomly selected. We assessed thresholds for pain tolerance before and 0, 3, 5, and 10 minutes after the cold pressor test (CPT). The response to the test stimulus was the pressure pain tolerance threshold (PPTT) measured at the ipsilateral 2nd toe, before and immediately after conditioning stimulus (CPT), which was applied to the contralateral hand. CPM was calculated as the absolute difference of pressure tolerance thresholds after-before CPT.

\section{Pressure Stimulation}

The test was applied at the center of the pulp of the 2nd toe. PPTT was measured with an electronic pressure algometer (Somedic, Hörby, Sweden). The pressure was increased from 0 at a rate of $30 \mathrm{kPa} / \mathrm{s}$ to a maximum pressure of $1000 \mathrm{kPa}$. PPTT was defined as the point at which the participant felt pain as intolerable. The participants were instructed to press a button when this point was reached. The algometer displays the pressure intensity at which the button is pressed. If patients did not press the button at $1000 \mathrm{kPa}$, this value was considered as pain tolerance threshold, although the tolerance level has not been reached.

At the beginning, the test was performed several times at the 2nd ipsilateral finger for training purposes until the participant was familiar with the procedure. These measurements were not used for the data analysis. Then, 3 assessments for
PPTT were made at the 2nd toe, and the average of these 3 measurements was calculated for the analysis of PPTT.

\section{Cold Pressor Test}

The contralateral hand was immersed in ice saturated water $\left(0.7 \pm 1{ }^{\circ} \mathrm{C}\right)$. Perceived pain intensity was continuously rated with an electronic VAS coupled to a pen recorder. Participants were instructed to keep their hand in the cold water for a maximum of 2 minutes. The device consisted of a container separated by a mesh screen into an outer and an inner part. The mesh screen prevents direct contact between the ice (placed in the outer part) and the hand of the participant (placed in the inner part). The water was regularly stirred to maintain the temperature in the inner part near to $0.7^{\circ} \mathrm{C}$, as monitored by a thermometer with a digital display $\left( \pm 0.1^{\circ} \mathrm{C}\right)$. Patients placed their hand, wide open and up to the wrist, into the inner part of the container. They were asked to keep it under water for a maximum time of 2 minutes. If pain was intolerable before 2 minutes had elapsed, the participant could withdraw the hand and the elapsed time was noted. The area under the curve of pain intensity over the 2-minute period was calculated.

\section{CPM}

At the very same time as the hand was withdrawn from the ice water, a single assessment of PPTT at the 2nd toe was made as described above. The difference in PPTT after-before the CPT was then calculated and considered as a measure of CPM. Positive values would indicate increase in PPTT and, therefore, functioning endogenous pain inhibition. This parameter, recorded immediately after hand withdrawal (time 0), was the primary outcome of this study. In addition, PPTT was measured 3, 5, and 10 minutes after the CPT, to analyze the time course of CPM.

\section{Statistical Analysis}

\section{Characteristics of Participants}

The 3 groups were compared using the $\chi^{2}$ test for the binary variable and 1-way analysis of variance for continuous variables.

\section{Primary and Secondary Outcomes}

Our primary hypothesis was that pain inhibition differs between the 2 groups of LBP patients and pain-free controls immediately after the CPT. The secondary hypothesis was that CPM diminishes faster in the 2 patient groups than in the control group.

We tested these hypotheses in a repeated measure linear-mixed model using restricted maximum likelihood estimation. The PPTT measurement immediately, ?3, 5, and 10 minutes after the CPT was defined as dependent variable, the patient group category and time-point category as independent variables. To study differences in the decrease of CPM between groups over time, we introduced interaction terms between groups and time-points. The model was adjusted for the baseline value of PPTT, withdrawal time out of the cold water, age, sex, and body mass index. To account for the correlation of repeated measures within participants, we introduced a random effect at the participant level and applied a within-participant autoregressive (AR 1) covariance structure. As we control the outcome variable (PPTT measured after the CPT) for the baseline 
PPTT measured before the CPT, we actually model differences in PPTT before and after the cold water test, that is, the CPM. Therefore, the resulting beta-coefficients can directly be interpreted as effects on CPM.

\section{Sample Size Considerations}

Sample size considerations were based on the inhibitory effect of the CPT on the PPTT, assuming a SD of the inhibitory effect of $100 \mathrm{kPa}$ in all 3 groups. A sample size of 30 participants per group provides a power of $87 \%$ to detect a difference in inhibition of $80 \mathrm{kPa}$ at a 2 -sided alpha of 0.05. All analyses were performed using Stata, Release 12 (Stata Corp LP, College Station, TX).

\section{RESULTS}

During the period of data collection of 40 patients with acute LBP, 34 chronic LBP patients, and 30 pain-free controls were recruited. Data were complete for all participants.

\section{Baseline Characteristics and Descriptive Variables}

Patients with acute and chronic LBP had a mean duration of pain of $1.8( \pm 1.0)$ weeks and $84( \pm 105)$ months, respectively. The mean pain intensity on a numeric rating scale from 0 to 10 was $5.2( \pm 1.5)$ for patients with acute LBP and on a VAS from 0 to 10 was $5.1( \pm 1.4)$ for chronic LBP patients. Demographic characteristics are reported in Table 1 . The groups differed only in age, with the chronic LBP group being older than the other 2 groups.

PPTT at all time-points and withdrawal time of the CPT are reported in Table 2 . The PPTT varied significantly among all groups at all the time-points, with higher values in pain-free controls compared with both patient groups (Table 2 and Figure 1). Withdrawal time from ice water did not vary significantly among groups $(P=0.30)$. The percent of participants who reached the pain tolerance to the CPT before the 2-minute limit was 87.5, 85.2, and 83.3 in the acute LBP, chronic LBP, and control groups, respectively.

\section{Primary Aim}

The mean difference in PPTT between measurement made immediately after and before the CPT (inhibitory effect) was: $164 \mathrm{kPa}(95 \%$ confidence interval [CI], 120-207) in acute LBP, $161 \mathrm{kPa}(95 \% \mathrm{CI}, 93-228)$ in chronic LBP, and $132 \mathrm{kPa}(95 \% \mathrm{CI}, 91-173)$ in controls (Fig. 2). A repeated measures mixed model did not show any explanatory effect of patient group on CPM immediately after the CPT as compared with the control group. The effect on CPM was $15.7 \mathrm{kPa}(95 \% \mathrm{CI},-51.6$ to $83.1 ; P=0.65)$ in chronic LBP and $-5.6 \mathrm{kPa}(95 \% \mathrm{CI},-69.2$ to 58.0 ; $P=0.83$ ) in acute LBP patients (Table 3 ).

\section{Secondary Aims}

The inhibitory effect decreased with time. Three minutes after the CPT, the values were $67 \mathrm{kPa}(95 \% \mathrm{CI}, 35$ $100)$ in acute LBP, $65 \mathrm{kPa}(95 \% \mathrm{CI}, 14-115)$ in chronic LBP, and $103 \mathrm{kPa}$ (95\% CI, 67-139) in controls (Fig. 2). Three minutes after the CPT, patients with acute LBP showed a significantly smaller CPM than controls (adjusted effect of $-72.6 \mathrm{kPa} ; 95 \% \mathrm{CI},-136.2$ to $-9.0 ; P=0.03$ ). The CPM was also smaller in patients with chronic LBP than in controls, but this effect was not statistically significant: $-50.9 \mathrm{kPa}(95 \% \mathrm{CI},-118.2$ to $16.5 ; P=0.14)$. There were no significant explanatory effects of groups on CPM 5 and 10 minutes after the CPT.

The time course of the inhibitory effect is illustrated in Figure 2. According to the repeated measures mixed model, patients with acute and chronic LBP exhibited a significantly steeper decline of the inhibitory effect between 0 and 3 minutes than pain-free patients, meaning that the inhibitory effect diminished faster in both patient groups compared with the control group (effect on decline in chronic LBP patients compared with controls: $-66.7 \mathrm{kPa}$; $95 \% \mathrm{CI},-119.9$ to $-13.5 ; P=0.01$; in acute LBP patients compared with controls: $-67.0 \mathrm{kPa} ; 95 \% \mathrm{CI},-118.3$ to $-15.7 ; P=0.01$; Table 3 ).

We additionally tested for associations between inhibitory effects at the time immediately after the CPT and pain duration as well as pain intensity in both patient groups. We found only weak and mainly nonsignificant correlations, suggesting that patients with longer lasting and more intense pain might exhibit smaller inhibitory effects (Figs. 3, 4).

\section{DISCUSSION}

\section{Main Findings}

In the present study, both patient groups and controls displayed a CPM effect, as PPTTs increased after CPT. There was no overall difference in CPM among the groups immediately after CPT, when calculated as difference afterbefore or as percent of change. However, the decline in CPM effect was significantly faster in chronic and acute LBP patients than in controls, which indicates that the endogenous inhibitory system can be less efficient in chronic and acute LBP patients. There was no evidence to suggest that acute and chronic LBP patients differed in their CPM efficacy.

\section{CPM in Low Back Pain}

Previous studies have found hyperalgesia and reduced endogenous pain modulation in chronic pain, as assessed by mechanical ${ }^{7-9}$ or thermal ${ }^{10}$ experimental pain modalities. This has been detected in different chronic pain conditions, such as osteoarthritis, ${ }^{7,9}$ irritable bowel syndrome, or

TABLE 1. Demographic Characteristic of Participants

\begin{tabular}{|c|c|c|c|c|}
\hline & $\begin{array}{l}\text { Acute Low Back Pain } \\
\qquad(n=40)\end{array}$ & $\begin{array}{l}\text { Chronic Low Back Pain } \\
\qquad(\mathrm{n}=34)\end{array}$ & $\begin{array}{l}\text { Controls } \\
(\mathrm{n}=30)\end{array}$ & $\boldsymbol{P}$ \\
\hline Age & $41.4(12.5)$ & $50.8(14.0)$ & $37.4(10.9)$ & $<0.001$ \\
\hline Sex (females) (n [\%]) & $16(40)$ & $17(50)$ & $16(53)$ & 0.499 \\
\hline BMI & $24.2(3.3)$ & $26.1(4.1)$ & $25.1(3.6)$ & 0.077 \\
\hline
\end{tabular}

Age and BMI are presented as mean (SD). Sex is presented as number (\%) of females.

BMI indicates body mass index $\left(\mathrm{kg} / \mathrm{m}^{2}\right)$. 


\begin{tabular}{|c|c|c|c|c|}
\hline & $\begin{array}{l}\text { Acute Low Back Pain } \\
(\mathrm{n}=\mathbf{4 0})\end{array}$ & $\begin{array}{l}\text { Chronic Low Back Pain } \\
(\mathrm{n}=\mathbf{3 4})\end{array}$ & $\begin{array}{l}\text { Controls } \\
(n=30)\end{array}$ & $\boldsymbol{P}$ \\
\hline \multicolumn{5}{|c|}{ Pressure pain tolerance threshold $(\mathrm{kPa})$} \\
\hline Before CPT & $388.6(136.3)$ & $407.8(178.6)$ & $548.8(183.6)$ & $<0.001$ \\
\hline $0 \mathrm{~min}$ after & $552.2(182.7)$ & $568.5(238.3)$ & $681.0(190.6)$ & 0.025 \\
\hline $3 \mathrm{~min}$ after & $456.0(144.3)$ & $472.7(195.7)$ & $651.8(202.1)$ & $<0.001$ \\
\hline 5 min after & $460.3(138.5)$ & $464.1(211.1)$ & $628.7(195.5)$ & $<0.001$ \\
\hline 10 min after & $450.0(140.8)$ & $473.3(223.5)$ & $601.5(188.5)$ & 0.003 \\
\hline \multicolumn{5}{|l|}{ Cold pressor test } \\
\hline Tolerance time (s) & $49.6(34.3)$ & $53.9(36.8)$ & $62.8(33.4)$ & 0.296 \\
\hline $\operatorname{AUC}(\mathrm{mm} \times \mathrm{s})$ & $9460(1522)$ & $8833(2427)$ & $8797(2038)$ & 0.284 \\
\hline
\end{tabular}

temporomandibular disorders. ${ }^{10}$ Hyperalgesia can predict a large proportion of the variance of clinical pain intensity in chronic musculoskeletal pain patients. ${ }^{11}$

The literature on CPM in chronic LBP is sparse. A recent review ${ }^{12}$ found only 1 study, not supporting an alteration in CPM. ${ }^{13}$ This confirms our assumption that measuring the time course may provide more information than single assessments.

Few studies have been performed on CPM in acute pain, and we are not aware of investigation on CPM in acute LBP. Interestingly, our results indicate that an alteration in endogenous modulation is likely present already in the acute phase of LBP. We did not find evidence for a more severe alteration of CPM in chronic LBP, indicating that changes in endogenous modulation may be equally important in acute and chronic pain conditions. The mechanisms underlying dysfunctional CPM are difficult to investigate in humans. Studies that correlate CPM with brain processes have revealed modifications in the activation of different brain areas ${ }^{14}$ and in the functional connectivity of the descending inhibitory system. ${ }^{15}$ The origin of these changes, however, remains unclear.

\section{Clinical Significance of CPM}

An intriguing question is whether those patients displaying an alteration in CPM in the acute phase are more prone to develop chronic LBP. In a recent exploratory study, we found no evidence for an association between CPM, measured during the course of chronic pain, and intensity of chronic LBP at follow-up. ${ }^{16}$ Another study on

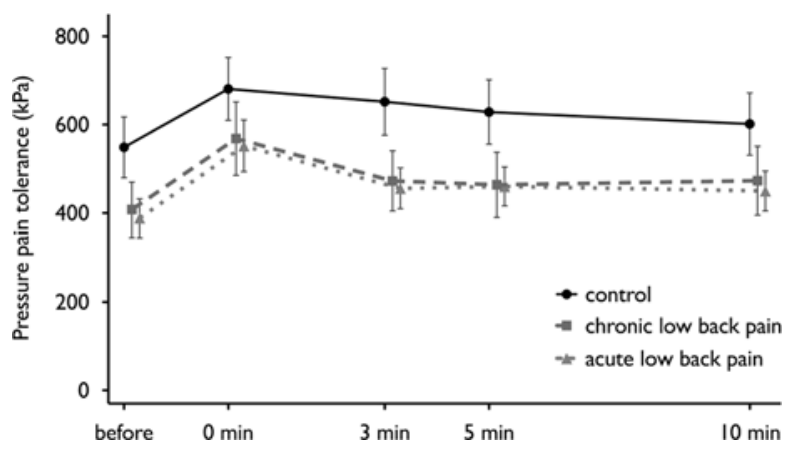

FIGURE 1. Pressure pain tolerance before $0,3,5$, and 10 minutes after the cold pressor test. The mean values with $95 \%$ confidence intervals are presented. acute LBP failed to demonstrate a predictive value of CPM for the development of chronic LBP. ${ }^{17}$ This is in contrast to the finding of a predictive value of CPM measures in chronic posthoracotomy pain. ${ }^{18}$ Possibly, we have not yet identified the type of CPM paradigm that most closely reflects alterations in central modulatory processes. Lack of information on the optimal methodology to assess CPM prevents conclusions on the prognostic ability of endogenous pain modulation.

Another important issue is the correlation between CPM and intensity of LBP. Obviously, pain intensity depends on many factors and CPM is not expected to explain a large part of the complex experience of pain. Few studies have addressed this issue. The most recent one did not find a statistically significant correlation between CPM and intensity of LBP; however, a composite score that included CPM and other parameters of pain sensitivity correlated significantly with pain intensity. ${ }^{19}$

It is important to note that both patient groups displayed an increase in PPTTs after conditioning stimulus; the mean differences between patients and controls were modest and not present at all time-points. Thus, we cannot make the generalized statement that patients with LBP have impaired endogenous modulation. It is more likely that a subset of patients display such altered central pain processing, but in the absence of normative data it is hard to identify these patients individually. Furthermore, our data do not support the view that altered CPM is a major determinant of hyperalgesia and pain in this patient population.

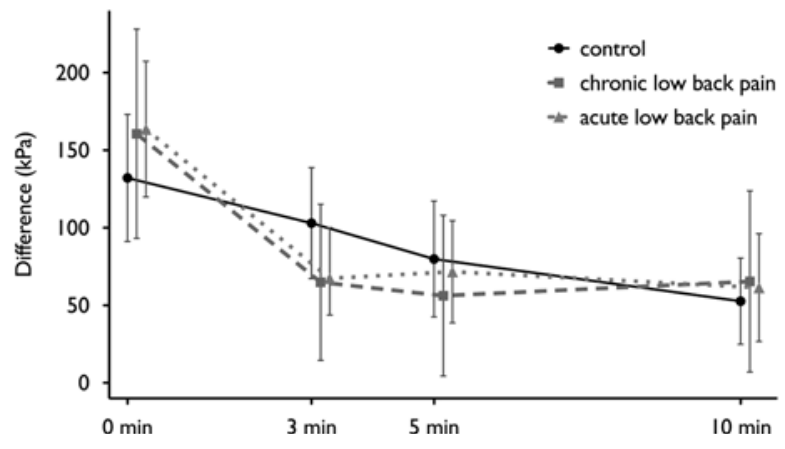

FIGURE 2. Pain modulation, calculated as the difference in pressure pain tolerance after-before cold pressor test at $0,3,5$, and 10 minutes. The mean values with $95 \%$ confidence intervals are presented. 
TABLE 3. Effects (Beta-coefficients) From a Repeated Measure Mixed Model With 95\% Confidence Intervals and $P$-values

\section{Effect on CPM \\ $(95 \% \mathrm{CI})(\mathrm{kPa})$ \\ $\boldsymbol{P}$}

Group (effects refer to the time-point immediately [0 min] after cold pressor test)

Pain-free controls

Chronic low back pain

Acute low back pain

Reference

$16.0(-51.4$ to 83.4$)$

$-5.5(-69.1$ to 58.1$)$

0.64

Time-point (effects refer to the control group)

0 min after

3 min after

Reference

5 min after

$-29.2(-67.9$ to 9.6$)$

$-52.3(-93.6$ to -11.0$)$

10 min after

$-79.5(-121.9$ to -37.2$)<0.00$

Interaction terms between group and time-points (effects refer to the difference in decline as compared with the control group)

Chronic low back $\quad-66.7(-119.9$ to -13.5$) \quad 0.01$

pain $\times 3$ min

Chronic low back

$-52.1(-108.9$ to 4.6$)$

pain $\times 5$ min

Chronic low back

$-15.7(-73.8$ to 42.4$)$

pain $\times 10 \mathrm{~min}$

Acute low back pain $\times 3$ min

Acute low back pain $\times 5$ min

Acute low back pain $\times 10 \mathrm{~min}$

Adjustment variables (centered)

Baseline PPTT (before

cold pressor test, per

$10 \mathrm{kPa})$

Withdrawal time from cold water (per $10 \mathrm{~s}$ )

Age (per decade)

Female sex

BMI (per unit)

Constant

$$
\begin{array}{ll}
-67.0(-118.3 \text { to }-15.7) & 0.01 \\
-39.6(-94.3 \text { to } 15.1) & 0.16 \\
-22.6(-78.6 \text { to } 33.4) & 0.43
\end{array}
$$

$$
8.1(6.9-9.4)
$$

BMI indicates body mass index; CI, confidence interval; CPM, conditioned pain modulation; PPTT, pressure pain tolerance threshold.

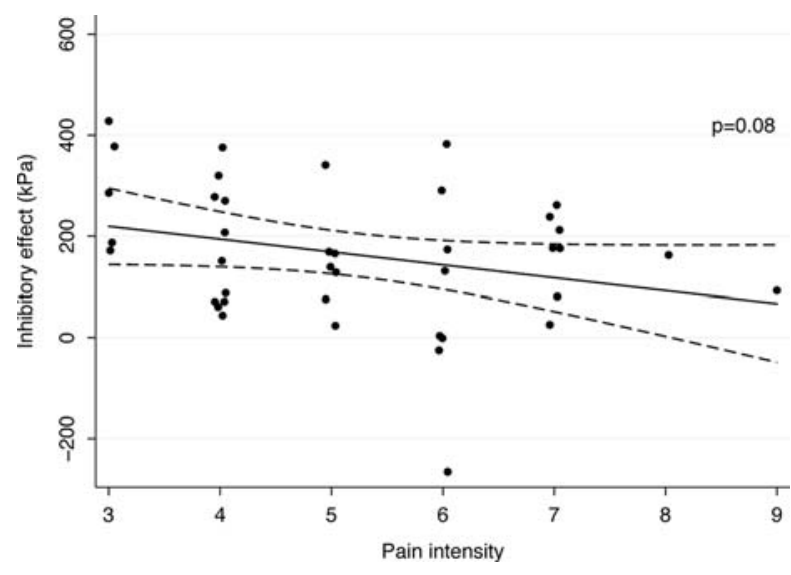

FIGURE 3. Association of the inhibitory effect immediately after the cold pressor test with pain intensity in acute low back pain patients. Scatter plot with a linear regression line, an associated $95 \%$ confidence interval, and $P$-value. The Spearman correlation is $-0.27(P=0.10)$. For the association with pain duration, the Spearman correlation is $-0.17(P=0.30$, graph not shown).

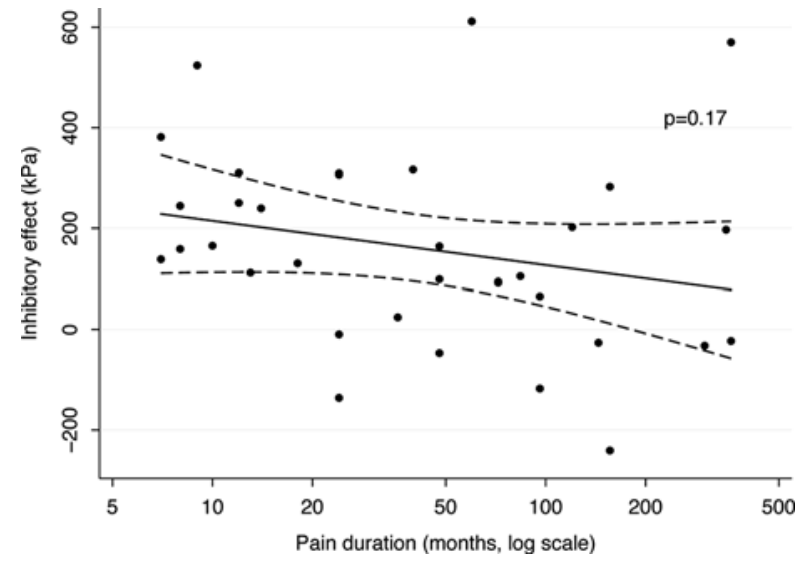

FIGURE 4. Association of the inhibitory effect immediately after the cold pressor test with pain duration in chronic low back pain patients. Scatter plot with a linear regression line, an associated 95\% confidence interval, and $P$-value. The Spearman correlation is $-0.35(P=0.046)$. For the association with pain intensity, the correlation is -0.08 ( $P=0.67$, graph not shown).

\section{Time Course of CPM}

In a recent review Pud et $\mathrm{al}^{2}$ concluded that the CPM effects fade gradually with time, and therefore simultaneous application of conditioning and test stimuli would be appropriate. In 1 study, the inhibitory effect on pressure pain thresholds after injection of hypertonic saline into the tibialis anterior muscle lasted for up to 15 minutes. ${ }^{20}$ Lewis et $\mathrm{al}^{21}$ found CPM to return to baseline after 15 minutes, using ice water or ischemic arm test as conditioning stimuli. In our investigation, there was no significant difference in CPM among the groups immediately after the CPT. If we had limited our testing procedure to an assessment immediately after cessation of the conditioning stimulus, we would have obtained only negative results. Our results therefore suggest that repeating the test stimulus over time can increase the chance of detecting alterations in endogenous pain modulation.

\section{Limitations of the Study}

A limitation of this study is the difference in average age in the 3 groups. However, the analysis was adjusted for this factor, limiting the likelihood of biased results. The complete time course of CPM is unclear, as the PPTTs were still elevated at the last assessment, made at 10 minutes. Because of the lack of a control session within groups, it is difficult to evaluate the contribution of habituation to the CPM effect. We evaluated CPM using only 1-test modality, that is, the CPT on the upper arm. We cannot rule out that using other methods would lead to different results.

In comparison with other studies we used temperatures around $0.7^{\circ} \mathrm{C}$ for our ice water test. The low temperature can be explained by the method of producing the ice water. Here, it has to be taken into account, that diffuse noxious inhibitory control should be more efficient the lower the temperatures are. $^{22}$ Further a maximum of pain could not be achieved within 2 minutes in some patients. A limit of this study is, that the method of producing ice water is not comparable with other studies and therefore cannot be used for meta-analysis.

\section{Conclusions}

In conclusion, the present study indicates a shorter duration of endogenous pain inhibition in patients with 
acute or chronic LBP as compared with controls. CPM was still detected in both patient groups, indicating that endogenous modulation, although effective for a shorter duration, is functioning at least partially. Accordingly, the relevance of diminished endogenous pain control is likely to vary greatly across patients. This stresses the need for developing tools to detect altered endogenous modulation in individual patients.

\section{REFERENCES}

1. Woolf CJ, Salter MW. Neuronal plasticity: increasing the gain in pain. Science. 2000;288:1765-1769.

2. Pud D, Granovsky Y, Yarnitsky D. The methodology of experimentally induced diffuse noxious inhibitory control (dnic)-like effect in humans. Pain. 2009;144:16-19.

3. Giesecke T, Gracely RH, Grant MA, et al. Evidence of augmented central pain processing in idiopathic chronic low back pain. Arthritis Rheum. 2004;50:613-623.

4. Laursen BS, Bajaj P, Olesen AS, et al. Health related quality of life and quantitative pain measurement in females with chronic non-malignant pain. Eur J Pain. 2005;9:267-275.

5. O'Neill S, Manniche C, Graven-Nielsen T, et al. Generalized deep-tissue hyperalgesia in patients with chronic lowback pain. Eur J Pain. 2007;11:415-420.

6. Neziri AY, Curatolo M, Limacher A, et al. Ranking of parameters of pain hypersensitivity according to their discriminative ability in chronic low back pain. Pain. 2012;153:2083-2091.

7. Arendt-Nielsen L, Nie H, Laursen MB, et al. Sensitization in patients with painful knee osteoarthritis. Pain. 2010;149:573-581.

8. Kosek E, Hansson P. Modulatory influence on somatosensory perception from vibration and heterotopic noxious conditioning stimulation (hncs) in fibromyalgia patients and healthy subjects. Pain. 1997;70:41-51.

9. Kosek E, Ordeberg G. Lack of pressure pain modulation by heterotopic noxious conditioning stimulation in patients with painful osteoarthritis before, but not following, surgical pain relief. Pain. 2000;88:69-78.

10. King CD, Wong F, Currie T, et al. Deficiency in endogenous modulation of prolonged heat pain in patients with irritable bowel syndrome and temporomandibular disorder. Pain. 2009; 143:172-178.
11. Staud R, Weyl EE, Price DD, et al. Mechanical and heat hyperalgesia highly predict clinical pain intensity in patients with chronic musculoskeletal pain syndromes. J Pain. 2012;13: 725-735.

12. Roussel NA, Nijs J, Meeus M, et al. Central sensitization and altered central pain processing in chronic LBP: fact or myth? Clin J Pain. 2013;29:625-638.

13. Julien N, Goffaux P, Arsenault P, et al. Widespread pain in fibromyalgia is related to a deficit of endogenous pain inhibition. Pain. 2005;114:295-302.

14. Wilder-Smith $\mathrm{CH}$, Schindler D, Lovblad K, et al. Brain functional magnetic resonance imaging of rectal pain and activation of endogenous inhibitory mechanisms in irritable bowel syndrome patient subgroups and healthy controls. Gut. 2004;53:1595-1601.

15. Jensen KB, Loitoile R, Kosek E, et al. Patients with fibromyalgia display less functional connectivity in the brain's pain inhibitory network. Mol Pain. 2012;8:32.

16. Mlekusch S, Schliessbach J, Camara RJ, et al. Do central hypersensitivity and altered pain modulation predict the course of chronic low back and neck pain? Clin J Pain. 2013;29: 673-680.

17. LeResche L, Turner JA, Saunders K, et al. Psychophysical tests as predictors of back pain chronicity in primary care. $J$ Pain. 2013;14:1663-1670.

18. Yarnitsky D, Crispel Y, Eisenberg E, et al. Prediction of chronic post-operative pain: pre-operative dnic testing identifies patients at risk. Pain. 2008;138:22-28.

19. O'Neill S, Manniche C, Graven-Nielsen T, et al. Association between a composite score of pain sensitivity and clinical parameters in low-back pain. Clin J Pain. 2014;30: 831-838.

20. Graven-Nielsen T, Babenko V, Svensson P, et al. Experimentally induced muscle pain induces hypoalgesia in heterotopic deep tissues, but not in homotopic deep tissues. Brain Res. 1998;787:203-210.

21. Lewis GN, Heales L, Rice DA, et al. Reliability of the conditioned pain modulation paradigm to assess endogenous inhibitory pain pathways. Pain Res Manag. 2012;17:98-102.

22. Wijk G, Veldhuijzen D. Perspective on diffuse noxious inhibitory controls as a model of endogenous pain modulation in clinical pain syndromes. J Pain. 2010;11:408-419. 